\title{
A Study on the Determination of Azithromycin by High-Performance Liquid Chromatography
}

\author{
Yanli Zhuo* \\ Shenyang Institute for Food and Drug Control, Shenyang 110000, Liaoning Province, China \\ *Corresponding author: Yanli Zhuo, zhuoyanli66@sina.com
}

\begin{abstract}
Objective: To analyze the effect of high-performance liquid chromatography (HPLC) for the determination of azithromycin and to provide references for related research work. Methods: The mobile phase was ammonium dihydrogen phosphate at $0.067 \mathrm{~mol} / \mathrm{L}$ (mixed with triethylamine; $\mathrm{pH}$ value was adjusted to 6.5 ). The chromatographic column was Kromasil C18 $(250 \mathrm{~mm} \times 4.6 \mathrm{~mm} ; 5.0 \mu \mathrm{m})$ and the relative standard deviation (RSD) of the drug content level was $1.25 \%$. The injection volume was set to $20 \mu \mathrm{L}$, the detection wavelength was set to $210 \mathrm{~nm}$, the external standard method was used to complete the quantitative work, and the theoretical plate number should be more than 1000 according to the drug peak calculation. The effect of HPLC on the determination of azithromycin was analyzed. Results: The concentration of azithromycin was $1.40-3.40 \mathrm{mg} / \mathrm{mL}$, and the linear relationship was good. RSD of the drug content level was $1.25 \%$. The representative test product had strong stability within 8.0 hours and the method had good repeatability. According to the recovery experiment method, the recovery rates of three standard samples from low to high were $99.87 \%, 100.15 \%$, and $100.62 \%$. The average recovery rate was $100.21 \%$. RSD value was $0.39 \%$. It means that the recovery rate of HPLC is good. Conclusion: In the determination of azithromycin, the use of HPLC to complete the work was of high sensitivity, simple, and fast. The method had good repeatability in the determination of drug components which is worthy of further promotion.
\end{abstract}

Keywords: Azithromycin; Composition determination; High-performance liquid chromatography

Publication date: July 2021; Online publication: July 30, 2021

\section{Introduction}

Azithromycin belongs to the second generation of macrolide antibiotics. It is able to achieve satisfactory results in the treatment of infectious diseases pertaining the reproductive and respiratory tract. Nowadays, azithromycin has been recommended as the first-line drug for the treatment of infectious diseases in the medical guidelines of many countries and regions. This drug is suitable for lower respiratory tract infections caused by sensitive bacteria ${ }^{[1]}$, upper respiratory tract infections caused by acute tonsillitis, pharyngitis, and rhinosinusitis ${ }^{[2]}$, simple genital infections caused by the protoplasm of Chlamydia, etc. In order to comprehensively analyze the determination of azithromycin by high performance liquid chromatography (HPLC) combined with the actual situation, this article analyzed the above proposition.

\section{Materials and methods}

\subsection{Baseline information}

The reference substance of azithromycin was selected, and the purity level was $95.05 \%$. The manufacturer was China Institute for The Inspection of Biological Products. Three batches of drug substances were selected: acetonitrile and methyl alcohol (chromatographic pure); triethylamine and ammonium dihydrogen phosphate (analytically pure) in which the manufacturer was Tianjin Damao Chemical Reagent Factory; self-made pure water. Kromasil C18 HPLC Column whose manufacturer was Dalian Elite Analytical 
Instrument Co., Ltd. was chosen.

\subsection{Methods}

\subsubsection{Preparation method of reference substance}

A standard precise weight of $25 \mathrm{mg}$ of azithromycin was placed it into a $50 \mathrm{ml}$ volumetric flask. The fluid phase was added to dissolve it and diluted to scale level. It was mixed well and prepared into a reference substance with a concentration of $0.5 \mathrm{mg} / \mathrm{mL}$.

\subsubsection{Preparation method of sample for test}

The drug substance was accurately weighed; $25 \mathrm{mg}$ azithromycin with the complete processing in accordance with 2.2.1. The test sample was prepared with a concentration of $0.5 \mathrm{mg} / \mathrm{mL}$.

\subsubsection{Drawing method of standard curve}

The reference drug was made into different concentration levels of 3.4, 3.0, 2.6, 2.2, $1.8,1.4 \mathrm{mg} / \mathrm{mL}$ solution. $20 \mu \mathrm{L}$ of solution was injected into the chromatograph and the peak area of the chromatogram was comprehensively recorded. The injection volume was regarded as the abscissa (X) and the peak area was regarded as the ordinate (Y). The curve was then drawn.

\subsubsection{Experimental chromatographic conditions}

The mobile phase was ammonium dihydrogen phosphate with the concentration of $0.067 \mathrm{~mol} / \mathrm{L}$ (mixed with triethylamine; $\mathrm{pH}$ adjusted to 6.5$)$. The chromatographic column was Kromasil $\mathrm{C} 18(250 \mathrm{~mm} \times 4.6$ $\mathrm{mm} ; 5.0 \mu \mathrm{m})$. The flow rate was set at $1.0 \mathrm{~mL} / \mathrm{min}$. Acetonitrile was 70:30, the injection volume was set to $20 \mu \mathrm{L}$, the detection wavelength was set to $210 \mathrm{~nm}$, the external standard method was used to complete the quantitative work, and the theoretical plate number should be more than 1000 according to the drug peak.

\subsubsection{Stability testing}

The same batch of bulk drug was taken, and the process was completed according to the test products. Place at room temperature, at 0.0 hour $(\mathrm{h}), 2.0 \mathrm{~h}, 4.0 \mathrm{~h}, 6.0 \mathrm{~h}$, and $8.0 \mathrm{~h}, 20 \mu \mathrm{L}$ of the solution was taken and placed into the liquid chromatograph to record the peak area and calculate the RSD value. The stability of the test product was analyzed.

\subsubsection{Repetitive experiment}

The same batch of bulk drug was taken, and the process was completed according to the test products. Liquid chromatography was injected 6 times, the peak area was recorded comprehensively, the RSD value was calculated, and the repeatability of the test method was analyzed.

\subsubsection{Sample testing method}

A total of 3 batches of bulk drug were used to prepare the test sample. $20 \mu \mathrm{L}$ of test sample and control sample were absorbed and injected into the liquid chromatograph to calculate the content of the drug.

\subsubsection{Recovery experiment}

A total of 9 drugs were taken from the same batch with 3 in each group. They were placed into the control solution of each concentration level, respectively to produce high, medium, and low levels of drug samples. The above samples were treated according to the test method and $20 \mu \mathrm{L}$ was injected according to the established chromatographic conditions. The peak area was recorded, and the drug content level was calculated. 


\section{Result}

\subsection{Drawing the standard curve}

In this experiment, the injection volume was regarded as the abscissa $(\mathrm{X})$ and the peak area as the ordinate (Y). A standard curve was drawn, an equation was obtained where $\mathrm{Y}=218.9000 \mathrm{x}-5.1904, \mathrm{r}=0.997(\mathrm{n}=$ 6) represented that the concentration of azithromycin was $1.40-3.40 \mathrm{mg} / \mathrm{mL}$, and the linear relationship was good.

\subsection{Results of sample chromatography}

The chromatographic data of the test and reference samples are detailed in Figure $\mathbf{1}$ and Figure 2.

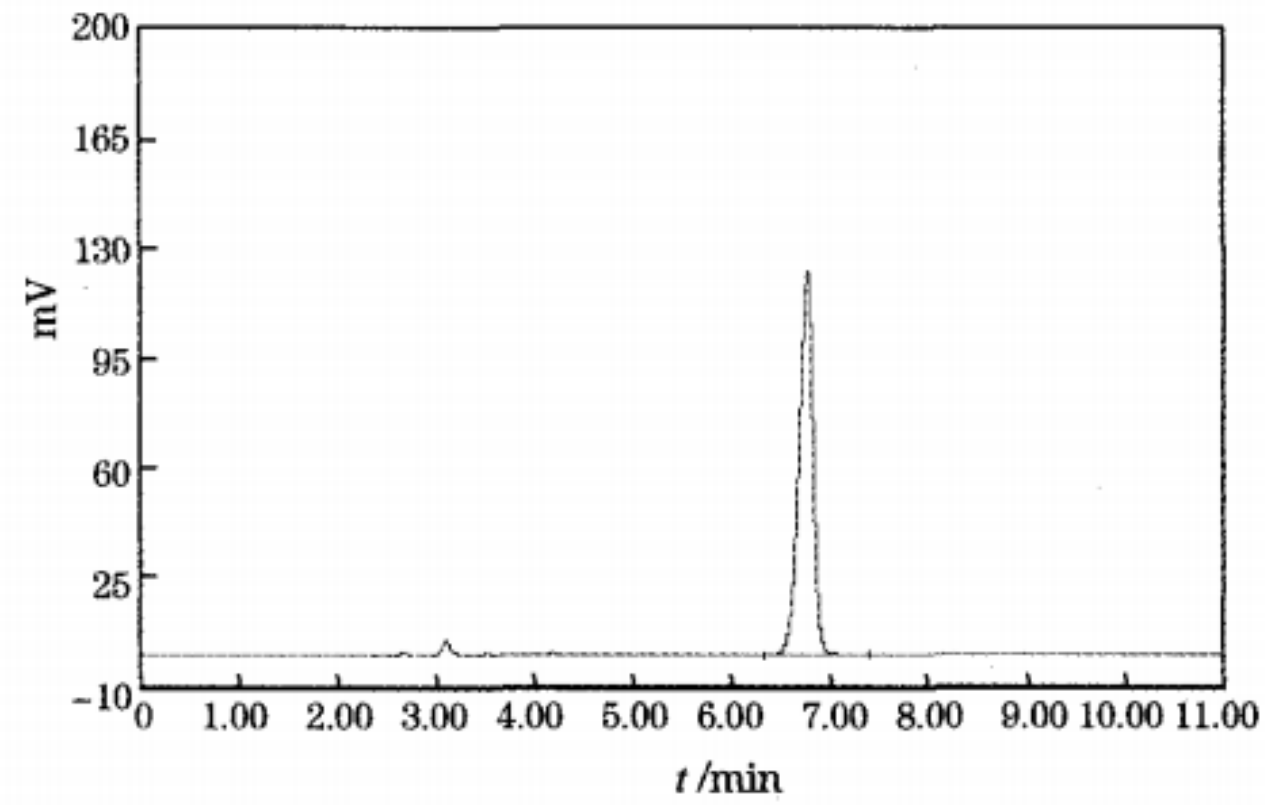

Figure 1. Chromatogram of test sample

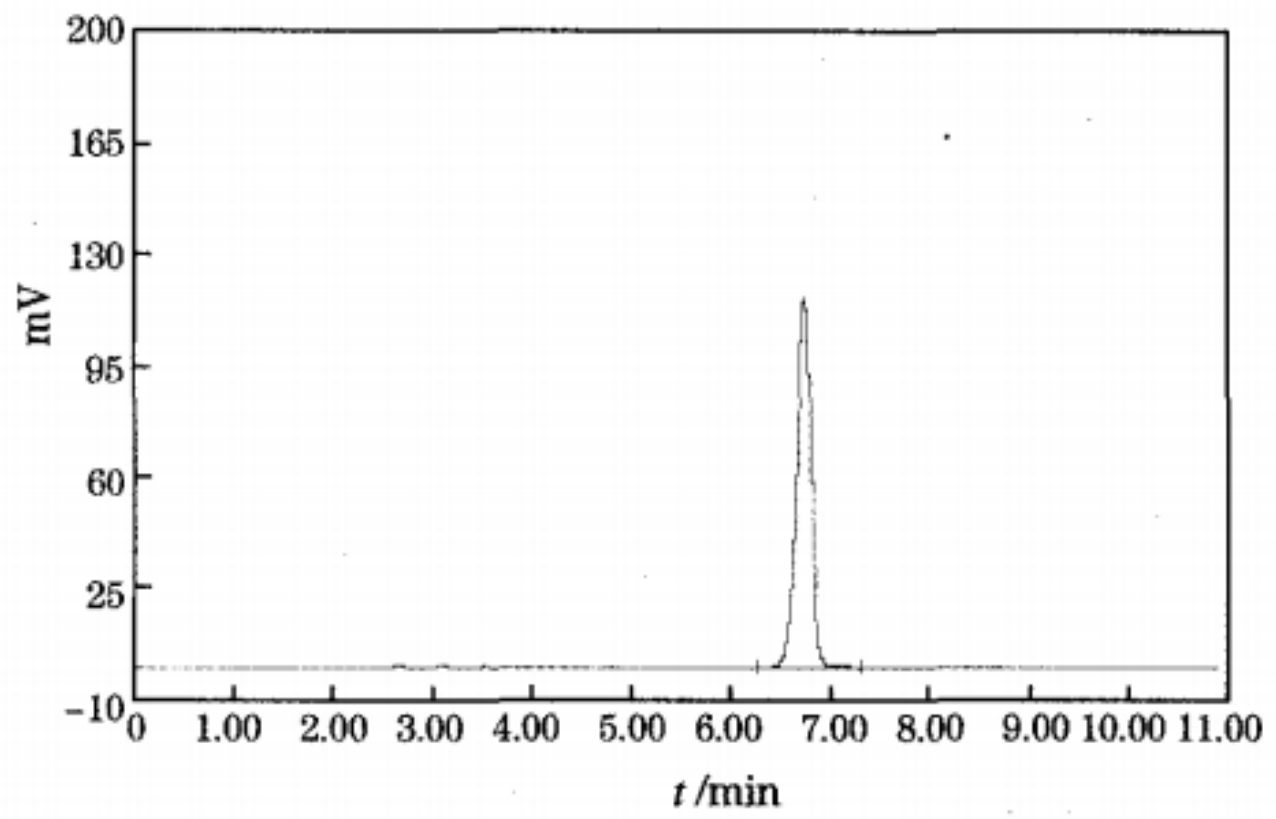

Figure 2. Chromatogram of reference substance 
It can be seen from Figure 1 and Figure 2 that the same drug peak appeared in the test sample at the retention time of the control sample. The results showed that this method was effective in the determination of azithromycin content.

\subsection{Results of stability test}

After the same batch of test samples were placed for different times, the determination results of the content level can be seen in Table 1.

Table 1. Determination results of drug content level of samples ( $x \pm s, \%)$

\begin{tabular}{cccc}
\hline Time & Drug content level & Degradation rate & RSD \\
\hline $0.0 \mathrm{~h}$ & $98.14 \pm 0.82$ & $0.00 \%$ & \\
$2.0 \mathrm{~h}$ & $97.83 \pm 0.33$ & $0.32 \%$ & $1.25 \%$ \\
$4.0 \mathrm{~h}$ & $96.34 \pm 0.22$ & $1.84 \%$ & \\
$6.0 \mathrm{~h}$ & $95.71 \pm 0.71$ & $2.49 \%$ & \\
$8.0 \mathrm{~h}$ & $95.54 \pm 0.51$ & $2.66 \%$ & \\
\hline
\end{tabular}

The RSD of content level was $1.25 \%$. The representative test product has strong stability within 8.0 hours.

\subsection{Repeatable experimental results}

Samples of the same batch were injected for a total of 6 times and the RSD value of the peak area was $0.50 \%$, indicating that this method had good repeatability.

\subsection{Sample determination}

A total of 3 batches of drugs were taken to prepare the test samples. $20 \mu \mathrm{L}$ of control samples and test samples were weighed, respectively and injected into the liquid chromatograph. External standard method and peak area were used to obtain the drug content level in test samples, as shown in Table 2.

Table 2. Determination results of drug content level in samples ( $x \pm \mathrm{s}, \%)$

\begin{tabular}{ccc}
\hline Sample batch & Drug content level & RSD \\
\hline$(1)$ & $94.80 \pm 0.41$ & $0.42 \%$ \\
$(2)$ & $93.75 \pm 0.12$ & $0.13 \%$ \\
$(3)$ & $93.95 \pm 0.25$ & $0.27 \%$ \\
\hline
\end{tabular}

\subsection{Recovery experiment}

According to the recovery experiment method, the recovery rates of three standard samples from low to high were $99.87 \%, 100.15 \%$, and $100.62 \%$. The average recovery rate was $100.21 \%$. RSD value was $0.39 \%$. This means that the recovery rate of high-performance liquid chromatography is good, and it is able to meet the requirements of related determination.

\section{Discussion}

High performance liquid chromatography (HPLC), also known as high-separation liquid chromatography or high-speed liquid chromatography belongs to the branch of total chromatography ${ }^{[3]}$. The high-pressure infusion system is used, and the mobile phase is liquid. Each polar solvent or each ratio of mixed solvent, 
buffer, and so on are pumped into the column. When the relevant components in the column are completely separated, they are put into the detector for detection. This method is used to complete sample analysis. Nowadays, HPLC is widely used in various fields.

At present, many literatures showed that the application of high-performance liquid chromatography in the determination of azithromycin has high accuracy, strong sensitivity, and simple operation ${ }^{[4]}$. In the determination of azithromycin, $\mathrm{CN}$ (cyano) column is used to complete the separation work in which satisfactory results can be obtained for the analysis and determination of drug components. In addition, it is worth noting that in the process of using $\mathrm{CN}$ column to separate azithromycin drugs, it can effectively reduce the proportion of organic solvents used to elute the drug mobile phase; hence, it can prevent column damage. When using short-wave UV to analyze the wavelength, the cost of organic solvent can be greatly reduced, and azithromycin can be eluted smoothly ${ }^{[5]}$.

For drugs and related substances, after spectral scanning by DAD (diode-array detection), end absorption can be found under the condition of drug chromatography. Therefore, the content of organic solvent must be effectively controlled in the specific mobile phase. In a literature, phosphoric acid was used to complete the adjustment in the chromatographic mobile phase, and the $\mathrm{pH}$ values were set to 3.0, 4.0, 5.0, 6.0, and 7.0. The influence of each $\mathrm{pH}$ value on the chromatographic column was analyzed. It was found that when the $\mathrm{pH}$ value of fluidity is 3.0-4.0, the theoretical number of trays meets the requirement of drug content determination. Azithromycin was determined by HPLC at $\mathrm{pH}$ values ranging from 3.0 to 3.5. In addition, it is worth noting that using sodium hydroxide and hydrochloric acid to prepare 6 kinds of different $\mathrm{pH}$ environment and after adjusting the $\mathrm{pH}$ value, the determination of azithromycin can be completed.

In this experiment, a total of two mobile phase systems had been selected. The first mobile phase was methyl alcohol and water in which the specific proportion was 80:20, respectively. However, the separation effect of this mobile phase was not satisfactory, and the retention effect was not good. After that, 0.067 $\mathrm{mol} / \mathrm{L}$ ammonium dihydrogen phosphate was added to acetonitrile in different proportions. The influence of this condition on the measurement results was analyzed. The results showed that when the proportion of acetonitrile was more than $30 \%$, the main peak and other miscellaneous peaks cannot be completely separated. When the proportion of acetonitrile was less than $25 \%$, the peak deformation of the main peak was blunt, showing significant trailing condition. The column efficiency was not good. Based on this situation, the concentration level of $0.067 \mathrm{~mol} / \mathrm{L}$ ammonium dihydrogen phosphate was considered to be applied and acetonitrile 70:30 was more suitable.

Because azithromycin belongs to the macrolide class of antibiotics and lacks chromogenic functional group, it would only show terminal absorption near 190-210 nm. Based on this, $210 \mathrm{~nm}$ was selected as the final detection wavelength in this experiment. This condition can be used to greatly improved the sensitivity of the correlation method.

In conclusion, in the determination process of azithromycin, the application of high-performance liquid chromatography to complete the work was of high sensitivity, simple, and fast. The method had good repeatability in the determination of drug components which is worthy of further promotion.

\section{Disclosure statement}

The author declares that there is no conflict of interest.

\section{References}

[1] Yu J, Rao J, Chen B, et al., 2009, Determination of Azithromycin and its Related Components by High Performance Liquid Chromatography Electrochemical Detection. Physical Testing and Chemical 
Analysis Part B: Chemical Analysis, 045(006): 636-8.

[2] Han Z, Li W, 2013, Study on the Determination of Azithromycin in Azithromycin Tablets by High Performance Liquid Chromatography. Tianjin Pharmacy, 25(001): 15-6.

[3] Cao J, 2019, Determination of Azithromycin by High Performance Liquid Chromatography. Northern Pharmacy, (10): 1-2.

[4] $\mathrm{Xu} \mathrm{N}, 2012$, The Pharmacokinetics and Relative Bioavailability of Azithromycin in Healthy Subjects were Studied by Liquid/Mass Spectrometry, National Conference on Clinical Pharmacology.

[5] Wang J, Bai D, Chen J, 2013, Determination of Azithromycin Sodium Dihydrogen Phosphate for Injection and Related Substances by HPLC, Chinese Pharmaceutical Journal Daojin Cup National Excellent Paper Selection Exchange Meeting, Pharmaceutical Analysis Committee of Chinese Pharmaceutical Association. 